\author{
Crossing the Border: International Journal of Interdisciplinary Studies \\ Volume 3; Number 1; 15 January 2015 \\ ISSN 2350-8752 (Print); ISSN 2350-8922 (Online)
}

\title{
ACUPUNCTURE AND MODERN MEDICINE
}

Asis De (India)

Abhijit De (India)

\begin{abstract}
It is estimated that acupuncture has been practiced in China for more than 4000 years. It has now gained increasing acceptance in many countries as an option for treatment of certain painful conditions. Chinese medicine works to balance the Qi (chi) energy in the body, balancing the body's female (yin) and male (yang) energies. Acupuncturists use needle to stimulate the 14 main meridians and up to 2000 points to prevent and treat diseases by regulating flow of chi through a person's energy channels within the body. There are five elements in acupuncture: fire, earth, metal, water and wood. They along with other six exogenous factors such as wind, cold, summer heat, damp, dryness and fire heat and miscellaneous pathogenic factors influence the energy flow through the body's five organ networks. According to WHO, acupuncture can be useful in pain management, organic lesions, substance abuse, gynecological disorders, neurological and psychiatric problems. Although it was originated in China, it has slowly spread through the years to Korea, Japan, Vietnam, Europe, and America. Different styles and modifications have been developed over the centuries and many of the countries have been integrating ancient Chinese medicine into modern medicine for treating patients. More importantly, it harmonizes our body energy, calms our mind and spirit and thereby enhances our capacity to enjoy life and attain happiness.
\end{abstract}

KEYWORDS: Acupuncture, Qi, Chinese medicine, Yang, Yin

\section{INTRODUCTION}

Acupuncture is an ancient way of healing and managing patients who have suffered from various illnesses. This technique was evolved in China long ago, more than 4000 years ago. Even today, several prehistoric human mummies (one of them is known as Ice Man), with well-preserved tattoos were discovered in Siberia, Peru, and Chile. Some of the tattoos were mystifying and archaeologists have no clue as to what those were for. Some suspected that these "tattoos" could be some kind of ancient acupuncture.

In China, acupuncture is still being practiced today. In the recent years, it has gained increasing acceptance in many countries as a treatment option for many painful conditions. It gained attention in the United States after President Nixon visited China in 1972. Mr. James Reston, a reporter with New York Times, was traveling with the presidential team who had to undergo an emergency appendectomy in China and was treated with acupuncture after his operation. Mr. Reston was so impressed with the post-operative pain relief by acupuncture that he wrote about this ancient Chinese medicine after returning back to the United States. ${ }^{1,2,3}$ 


\section{Crossing the Border: International Journal of Interdisciplinary Studies}

\section{PRINCIPLES OF CHINESE MEDICINE}

Chinese medical theory is based on the concept of Yin and Yang. Yin is considered feminine energy and Yang as masculine energy. Principles of Chinese Medicine are based on maintenance of the balance of the Qi (chi) energy in the body. Chinese medicine tries to keep the body's female (Yin) and male (Yang) energies balanced. The goal of each treatment is to increase depleted Yin or Yang or to eliminate excess Yin or Yang that keeps a proper balance between the two energies and maintains health of an individual.

Qi (pronounced chi) is considered as "life force energy" or "life activity." Chinese medicine believes that health is a function of a balanced, harmonious flow of that energy. The energy must be able to flow freely throughout the body in order to maintain overall health of a person. When there is a blockage or imbalance in the flow of Qi, the person falls sick. The pathway through which Qi flows is called meridians. The energy (Qi) circulates through these meridians to all the cells of our body.

Qi is the vital force, the presence of which separates the living from the dead. It's unbalanced or unimpeded flow is critical for good health. Qi flows through each one of us throughout our body like a river, energizing, nourishing and supporting every cell, tissue, muscle, organ and gland. Any blockage or derangement of the flow or imbalance of Qi will result in pain, dysfunction and ill health. These imbalances can be caused by stress, trauma, accidents, improper diet, strain, surgery, etc. Traditional Chinese medical technique for unblocking the flow of Qi is by acupuncture; when the fine needles are inserted at specific points on the body, which regulate the flow of Qi. These insertion points are believed to correspond with 14 main pathways or meridians within the body.

Traditional Chinese medicine describes the body's balance in terms of Yin and Yang. Yin is female energy that represents traditional female qualities of peacefulness and calm. It is hypoactive, which is inhibited, quiescent, shallow, pale and "female." They can be linked with fluids, water and damp. Yin syndrome is like someone depressed, tired and with lassitude. Yang on the other hand is male energy that represents traditional male qualities of aggression and stimulation. It is hyperactive, which is excited, fidgety, bright, red and "male". They can be linked with fire and movement. Yang syndrome is like someone anxious, agitated and having insomnia.

There are five elements in acupuncture: fire, earth, metal, water and wood. The energy that flows through us is influenced by these elements. The five elements support and build upon each other. Each of the body's five organ networks corresponds with a particular element.

- Heart/Small intestine with fire

- Spleen/Stomach with earth

- Lungs/Large intestine with metal

- Kidneys/Bladder with water

- Liver/Gallbladder with wood

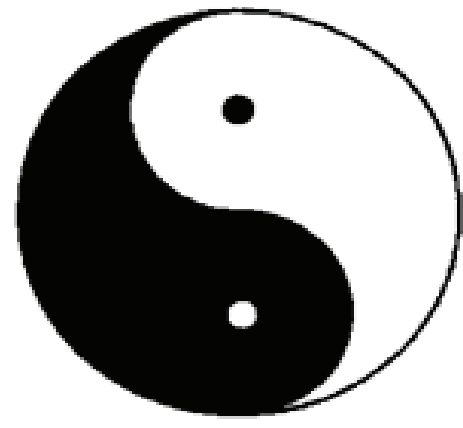

Figure 1: White = Yang Black $=$ Yin 


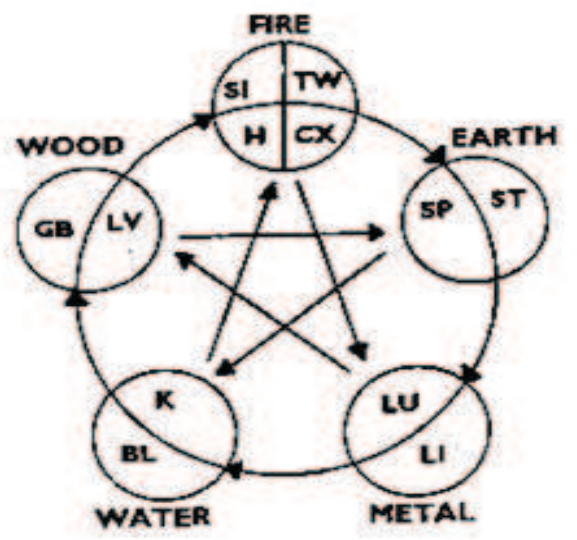
and unbalanced.

There are 12 main (6 pairs) of meridians, 2 unpaired (Du and Ren), and 8 secondary. 361 points plus extra points (up to 2000 points) along meridian complex regulate different areas of the body1,4,5 These points have numbers and names, standardised by World Health Organization (WHO) e.g. Sanyinjiao / Three Yin Junction / SP6; Fengchi / Pool of Evil Wind / GB20; Yingxiang / Welcome the Smell / LI20.

\section{Zang-Fu (Internal}

Organs): In traditional Chinese medicine the term refers to anatomical structures and physiological functions of the internal organs of the body as a whole.There is coordination in physiological activities and functional connection among Zang-Fu organs also with the sense organs and tissues in the body.

Zang (Yin): Solid e. g. lung, heart, spleen, kidney, liver, pericardium, (Conception vessel).

Fu (Yang): Hollow e. g. large intestine, small intestine, stomach, bladder, gallbladder (Sanjiao), (Governor vessel). 6

Some of the factors influence the health and the disease process as per the
Figure 2: Five Interacting Elements

Meridians: Meridians are invisible lines where the Qi (chi, life energy) flows. Different meridians correspond with specific organs or organ systems. The body has been mapped with these meridians that pass through organs or organ systems. Qi flows along the meridians of the body. Acupuncture needles are inserted in an attempt to balance the opposing forces of Yin and Yang. Yin and Yang are balanced when a person is healthy and Qi is there-
causes Yin and Yang to become distressed

Figure 3: Meridians

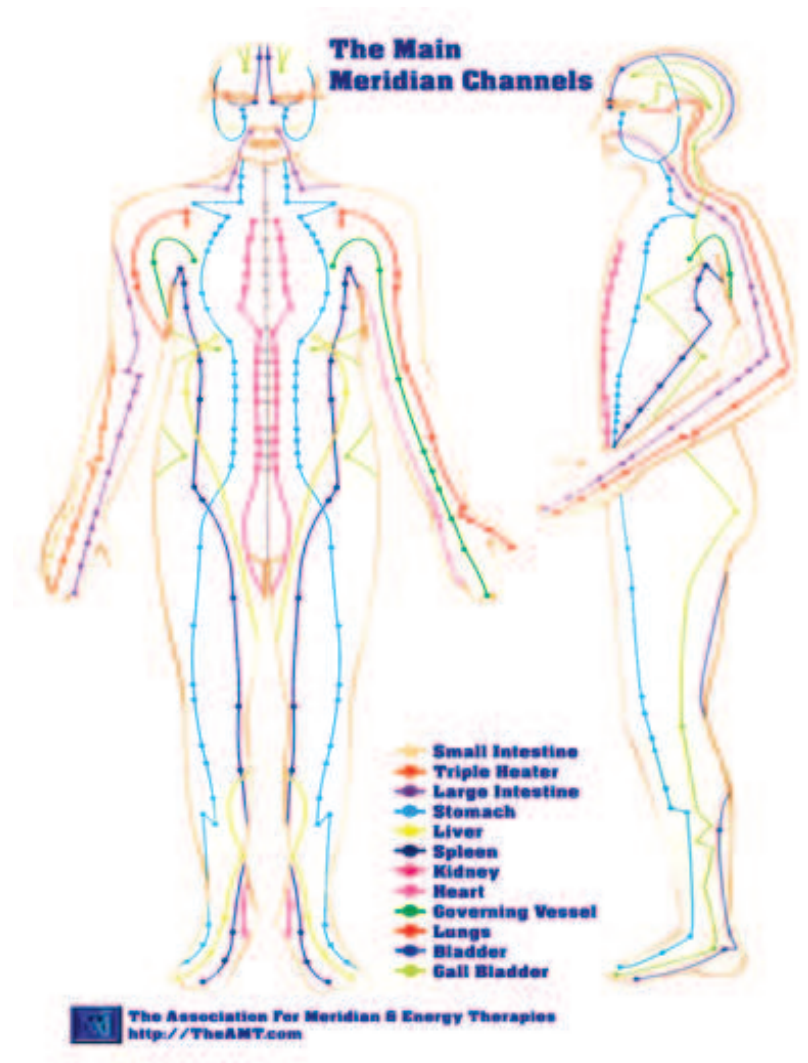




\section{Crossing the Border: International Journal of Interdisciplinary Studies}

ancient Chinese medicine are as follows:

\section{Six exogenous factors:}

1. Wind 2. Cold 3. Summer heat 4. Damp 5. Dryness 6. Fire heat

Miscellaneous pathogenic factors:

1. Irregular food intake 2. Stress 3. Lack of exercise 4. Traumatic injuries 5. Stagnant blood or phlegm 6. Meridian network system 7. Disruption of flow results in illness

Eight diagnostic principles:

1. Exterior 2. Cold 3. Deficiency 4. Yin 5. Interior 6. Heat 7. Excess 8. Yang ${ }^{1,4,5}$

\section{ACUPUNCTURE PROCEDURE}

Acupuncture, if practiced properly, can redirect and normalize the flow of Qi energy and bring the patient back to health. In acupuncture, hair thin, sterile, stainless steel needles $(35 \mathrm{G})$ are inserted into points along the meridian pathways. The needles are placed just below the surface of the skin in the epidermis. The needles are sometimes stimulated by twirling or heat moxibustion.

Fourteen major energy channels called meridians course through the human body including the head, arms, hands, feet, torso and internal organs.

Acupuncturists use needles to stimulate the meridians and points to prevent and treat diseases in order to regulate and unblock the person's energy channels. They always combine with other therapies.

Originally there were 365 acupuncture points, but through the years this number has increased to over 2,000. Each point is believed to correspond with a particular illness or injury. $1,4,5,7,8$

\section{Picture 4: Ancient Chinese chart of Acupuncture}

\section{MODE OF ACTION}

The effects of acupuncture are complex. How it works is not entirely clear. Research suggests that the needling process, and other techniques used in acupuncture, may produce a variety of effects in the body and the brain. One theory is that stimulated nerve fibers transmit signals to the spinal cord and brain, activating the body's central nervous system. The spinal cord and brain then release Meridians and Points
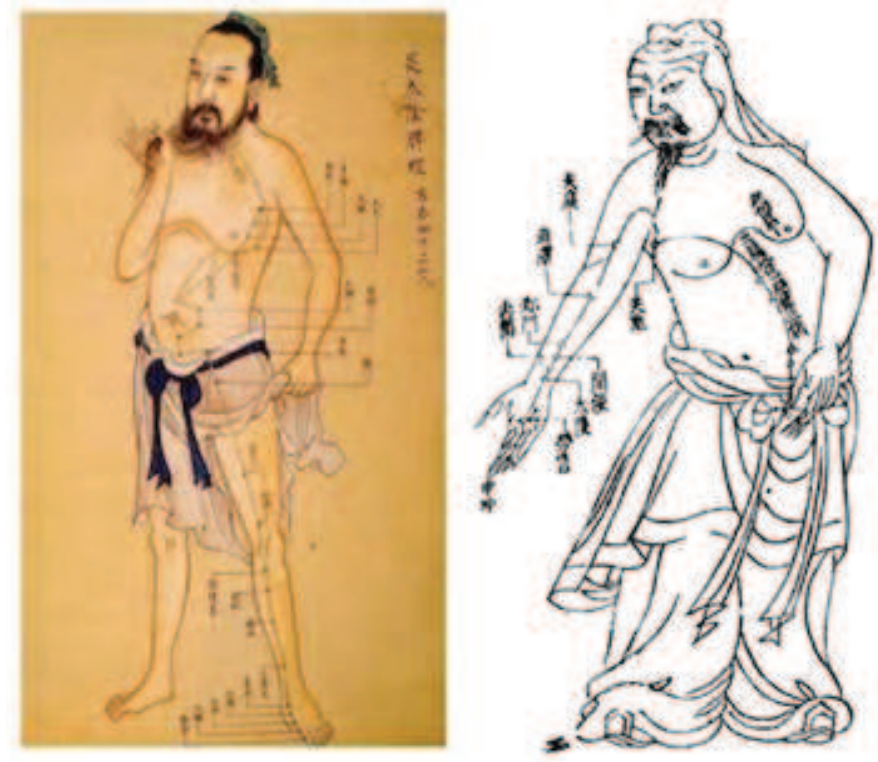

hormones responsible for making us feel less pain while improving overall health. In fact, a study using images of the brain confirmed that acupuncture increases our pain threshold, which may explain why it produces long-term pain relief. Acupuncture may also increase blood circulation and body temperature, affect white blood cell activity 


\section{ACUPUNCTURE AND MODERN MEDICINE}

(WBC is responsible for our body immunity), reduce cholesterol and triglyceride levels, and regulate blood sugar levels.

According to modern medicine, researches have shown that acupuncture causes release of endorphins. These studies also demonstrated that needling produces changes in the electrical conductivity of the skin. The exact mechanism of how acupuncture works is not known except that the needles stimulate the nervous system to release endorphins or other naturally occurring chemicals and hormones that affect mood, health and relieve pain. $1,4,7,8$

\section{DIFFERENT ACUPUNCTURE TECHNIQUES}

1. With Needles: After the acupuncture needle is inserted at the correct meridian point, there is a sensation of numbness, distension, or electrical tingling at the needling site which might radiate along the corresponding meridian which is called De-qi or "arrival of qi". In Chinese acupuncture, achieving De qi is an important part of the treatment.

2. Non-invasive needling: Acupressure is often described as "acupuncture without needles. It is a non-invasive form of acupuncture, uses physical pressure applied to acupressure points by fingers, the hand, elbow, or with various devices.

3. Electro-Acupuncture: It is a form of acupuncture in which acupuncture needles are attached to a device that generates continuous electric pulses to stimulate nerves.

4. Moxibustion: Traditionally, acupuncture is used to treat acute conditions while moxibustion is usually used for chronic diseased conditions by strengthening Yang and inducing smooth flow of Qi through the meridians. Burning cone or stick shaped preparations of moxa wool (made from a species of dried Chrysanthemum) is placed on or near an acupuncture point along with acupuncture to have a combined effect on the patient.

5. Cupping: In this ancient Chinese form of medicine, a local suction is created on the skin by placing hot cups made either from bamboo or glass over the skin at particular points to mobilize blood flow in order to Figure 5: Moxibustion promote free flow of Qi thereby diminishing swelling and pain.

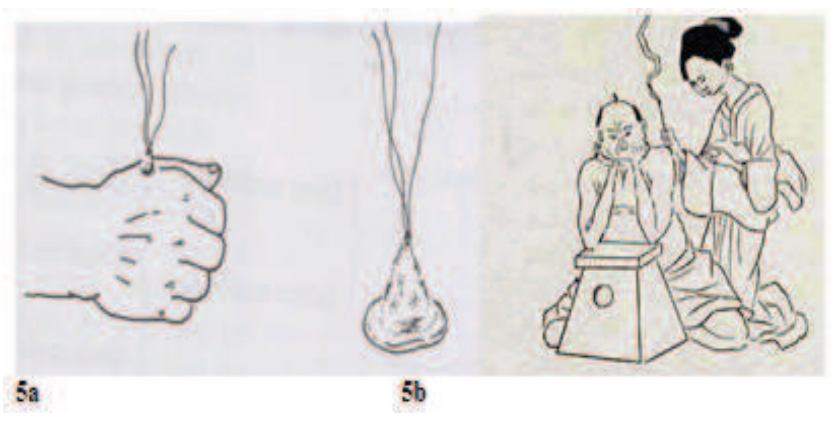

6. Tui Na: In this traditional Chinese medicine, the flow of Qi is regulated by attempting to stimulate different areas by various bare-handed techniques with out any needles.

7. Sonopuncture: It is a stimulation of acupuncture meridian points on the body using narrow ultrasound beam up to a depth of 6 to $8 \mathrm{~cm}$ instead of needles..$^{1,4,5,7,8,9,10}$ 


\section{Crossing the Border: International Journal of Interdisciplinary Studies}

\section{DIAGNOSIS OF PATIENT'S CONDITION}

An acupuncturist assesses tongue, skin, complexion, smell, pulse, body build, mental and emotional state of the patient. He/She also checks the pulse to assess the condition of all the organ networks and the flow of Qi through the twelve major meridians. The pulse is felt at six locations \& three depths on each wrist. The pulse is checked for its strength of each pulsation, its length, tailing off and any variation between pulsations. The three regions Cun, Guan and Chi of left hand reflect the conditions of heart, liver and Kidney and of the right hand reflect the conditions of the lungs, spleen and kidney. ${ }^{1,4,8}$

\section{TREATMENT}

Each acupuncture session is based on symptoms, pulse diagnosis and specific goals of treatment. Usually at each session, different points are needled. The needles may be inserted for a few seconds or may be left in situ for as long as 45 minutes. Sometimes the needles are attached to electric stimulator (Electro acupuncture) to stimulate the meridian points depending on the patient's condition and degree of stimulation required for treating the specific disorder. Pulses are checked again after the treatment. ${ }^{1,4,5,7,8,9}$

\section{RESULT OF TREATMENT}

A lot of questions are asked about the results of acupuncture. In a nationwide survey of acupuncture users in USA, 91.5\% reported "disappearance" or "improvement" of symptoms after acupuncture treatment, $84 \%$ said they need to visit their physicians less, $79 \%$ said they need less prescription medicines and $70 \%$ of those to whom surgery had been recommended said they could avoid surgery. ${ }^{10,11,12}$

\section{EFFECTIVENESS}

Some people might think that acupuncture has a placebo effect on the person having a disease or disorder. It is effective for some but not all conditions. It is mostly used for pain relief, nausea, vomiting, addiction (such as alcoholism), asthma, headaches, cramps, etc. ${ }^{1,2,13,14,15}$

Acupuncture in Surgical Patients: It was observed that the optimum time of acupuncture treatment was 2 days, 1 or 2 weeks after surgery. The patients after acupuncture had less swelling and pain, improved in mental alertness and there was improvement in intestinal mobility and urination. It shortened recovery time and promoted incision healing. ${ }^{16}$

Acupuncture for Cancer Patients: The optimum time of acupuncture treatment is 1 to 2 hours before treatment and 24 hours after treatment or only once before chemo therapy is given.

i. Radiation Therapy: The patients had less fatigue. It helped anemia, increased white blood cell count, reduced nausea, vomiting and diarrhea, decreased hair 


\section{ACUPUNCTURE AND MODERN MEDICINE}

loss and treatment induced infertility.

ii. Chemotherapy: Reduced nausea and vomiting, decreased hair loss, helped fatigue and shortness of breath, strengthened immune system, increased appetite,

helped red and white blood cell counts and neuropathy, and stopped diarrhea. ${ }^{17,18}$ According to World Health Organization, acupuncture can be useful in pain (chronic, surgical, arthritic, malignant, headache, and backache), organic lesions (cardiovascular, respiratory, GI, skin, and urological), neurological (peripheral \& central), substance abuse (drugs, nicotine, food and alcohol), gynecological disorders, psychiatric (depression and anxiety).19,20,21,22

In 1979, WHO listed over 40 conditions which can be treated by acupuncture:

- Eye-Ear-Nose-Throat - cataracts, gingivitis, poor vision, tinnitus, toothache, tonsillitis

- Digestive - abdominal pain, constipation, diarrhea, hyperacidity, indigestion

- Neurological - headaches, migraines, neurogenic bladder dysfunction, Parkinson's disease

- Musculoskeletal - arthritis, back pain, muscle cramping, muscle pain and weakness, sciatica

- Gynecological - infertility, menopausal symptoms, premenstrual syndrome

- Emotional - anxiety, depression, insomnia, nervousness, neurosis

- Miscellaneous - addiction control, athletic performance, blood pressure regulation, chronic fatigue, immune system enhancement, stress reduction, postoperative pain, stroke, asthma, bronchitis, common cold, sinusitis, smoking cessation. ${ }^{23}$

The U.S. National Institutes of Health (NIH) in 1997 recognized acupuncture as a mainstream medicine. It was also reported that acupuncture is effective in the treatment of: nausea, headache, dental pain, fibromyalgia, addictions, tennis elbow, asthma, stroke rehabilitation, osteoarthritis, menstrual cramps, myofascial pain, carpal tunnel syndrome, and lower back pain. ${ }^{24}$

\section{CONCLUSION}

Acupuncture originated in China but has spread to Korea, Japan, Vietnam, Europe, and America. Different styles have been developed over the centuries based on different ideas and techniques. Various medical institutions in many developed countries are carrying out researches combining modern medicine and various aspects of acupuncture for management of acute or chronic ailments and to alleviate pain and relief to the patient.

Acupuncture rejuvenates the normal function of meridians, regulates the function and balance of Yin / Yang, flow of Qi / Blood and Zang / Fu in our body systems. It, thus, improves our quality of life to enjoy better health and live in happiness.

\section{END NOTES}

1. Essentials of chinese acupuncture. (1980). Foreign Languages Press, Beijing, (1st Ed).

2. Ma, K.W. (1992). The roots and development of Chinese Acupuncture: From prehistory to early 20th Century. Acupuncture in Medicine, 10, 92-99. doi:10.1136/aim.10.Suppl. 92.

3. White, A., Ernst, E. (2004). A brief history of acupuncture. Rheumatology. (Ox ford, England): 43 (5): 662-663. 


\section{Crossing the Border: International Journal of Interdisciplinary Studies}

4. Dr. Bhabani Prasad Sahoo. (2005). Handbook on acupuncture treatment and research. Deep Prakashan (3rd Ed).

5. An outline of chinese acupuncture. (1975). Foreign Languages Press, Peking.

6. Ross, J. (1984). Zang Fu, the organ systems of traditional Chinese medicine. Else vier: 26.

7. Angela Hicks. (2005). The acupuncture handbook: How acupuncture works and how it can help you. Piatkus Books (1st Ed.): 41.

8. Bhabani Prasad Sahoo. (2004). Acupuncture. Mandal \& Sons (Book in Bengali).

9. Deadman, P., Baker, K, and Al-Khafaji, M. (2007). A manual of acupuncture. Journal of Chinese Medicine.

10. Maciocia, G, (2005). The foundations of Chinese medicine. Churchill; Livingstone.

11. Chinese acupuncture and moxibustion. (1987). Foreign Languages Press, Beijing.

12. Classics of traditional medicine. (2012). United States National Library of Medicine.

13. Steven Aung, and William Chen. (2007). Clinical introduction to medical acu puncture. Thieme.: 116.

14. Jin G., Jin, J.X., Jin, L.L. (2006). Contemporary medical acupuncture - A systems approach. Springer.

15. Flaws, B; Finney, D. (2007). A handbook of TCM patterns \& their treatments. Blue Poppy Press. (6th Ed.): 169-173

16. Lee, M. S., Ernst, E. (2014). Acupuncture for surgical conditions: an overview of systematic reviews. International Journal of Clinical Practice, 68(6):783-789.

17. Paley, C. A.; Johnson, M. I.;Tashani, O. A.; Bagnall, A. M. (2011). Acupuncture for cancer pain in adults. The Cochrane Database of Systematic Reviews, (1).

18. Garcia, M. K., McQuade, J., Haddad, R., Patel, S., Lee, R., Yang, P., Palmer, J. L., Cohen, L. (2013). Systematic review of acupuncture in Cancer care: A synthesis of the evidence. Journal of Clinical Oncology, 31(7): 952-60.

19. Mann, F. (2000). Reinventing acupuncture: A new concept of ancient medicine. Elsevier.

20. Schwartz, L. (2000). Evidence-based medicine and traditional Chinese medicine: Not mutually exclusive. Medical Acupuncture, 12(1): 38-41.

21. Ernst, E.; Pittler, MH; Wider, B; Boddy, K. (2007). Acupuncture: its evidencebase is changing. The American Journal of Chinese Medicine, 35(1): 21-25.

22. Johnson, M. I. (2006). The clinical effectiveness of Acupuncture for pain relief-you can be certain of uncertainty. Acupuncture in Medicine: Journal of the Brit ish Medical Acupuncture Society, 24(2): 71-79.

23. Ernst, E. (2006). Acupuncture--a critical analysis. Journal of Internal Medicine, 259(2): 125-137.

24. Sampson, W. (2005). Critique of the NIH Consensus Conference on Acupunc ture. Quack Watch.

\section{ABOUT THE AUTHORS}

Dr. Asis De is a Professor at Kathmandu University, Manipal College of Medical Sciences in Pokhara, Nepal who teaches community medicine. He studied acupuncture at Kolkata Acupuncture Medical College in 2007. Email: dr_asisde@hotmail.com

Dr. Abhijit De is a Medical Graduate from Kathmandu University, Manipal College of Medical Sciences, Pokhara, Nepal.Email: abhijitde7@gmail.com 